\title{
LA MÉTÉOROLOGIE, UNE DÉMARCHE PÉDAGOGIQUE POUR UNE ÉCOLE DE SENS
}

\author{
Pierre-Jean Cardona \\ École élémentaire Paul-Bert \\ 2, rue François-Mauriac \\ 89100 Sens
}

RÉSUMÉ

Dans cet article, un enseignant de l'École élémentaire montre comment il a utilisé des expériences simples de météorologie pour amener ses élèves (classes de CE2, CM1 et CM2) à la découverte de la Terre et à la pratique de la pensée expérimentale et rationnelle.

\section{ABSTRACT Meteorology as a teaching tool for a primary school in Sens}

In this paper, a teacher of the Primary School shows how he used simple experiments in meteorology to teach his pupils about the Earth and the practice of experimental and rational thought.

En décembre 1993, l'école Aristide-Briand, de Sens (Yonne), déposait, dans le cadre de la mise en place d'un contrat de ville, un projet de création d'un espace scientifique dans ses locaux. Située en Zone d'éducation prioritaire, c'està-dire dans un quartier où les familles ont souvent des difficultés sociales, l'école voulait offrir aux enfants les moyens de pouvoir pratiquer les sciences de façon sérieuse, en les abordant par la découverte et l'expérimentation.

Jusqu'ici, étant donné le recrutement de ses élèves, l'école avait surtout insisté sur les aspects de la maîtrise de la langue. Il était temps de mettre en route un processus qui, tout en poursuivant le travail et les efforts sur le français et le calcul, englobe en plus le domaine scientifique. Les activités de français (lecture, production de textes), lorsqu'elles sont considérées comme des activités transversales au cours de la mise en place d'activités scientifiques, sont en général bien acceptées par les élèves ; en effet, elles leur apparaissent dans ce cas comme des outils indispensables.

Notre projet s'appuyait, non seulement sur la didactique des sciences qui permettent de développer la compréhension de notre environnement, de la vie et de ses mécanismes, mais aussi, au-delà, sur le développement de la citoyenneté. En effet, tout en favorisant une pensée rationnelle, les activités scientifiques développent la rigueur, permettent de formuler des hypothèses, de les vérifier et donc d'opérer des choix. D'une façon plus générale, opérer des choix, en toute indépendance, quels qu'en soient les domaines, nous paraît un objectif fondamental dans l'éducation des enfants qui nous sont confiés.

Malheureusement, notre projet ne fut pas accepté dans le cadre de la mise en place du contrat de ville (qui aurait pu lui apporter un financement annuel durant cinq années). Il fallut donc le revoir à la baisse ; néanmoins, fin juin 1996, un espace « scientifique » fut inauguré à l'école de façon très officielle. Situé dans 
deux salles au cœur même de l'école, il fut utilisé par certaines classes dès l'année scolaire 1995-1996. Afin d'atteindre l'objectif fixé, être, pour les enfants, un lieu de découverte incitatif à la recherche et à la pratique d'activités scientifiques et techniques, il se veut être un centre de ressources où les élèves peuvent découvrir, expérimenter, développer leur curiosité, avoir une attitude active et critique. À cet effet, les salles sont aménagées autour de cinq grands thèmes :

- le vivant (élevage de poissons et d'insectes dans un premier temps),

- les cinq sens,

- la physique (électricité, mécanique),

- la robotique (pilotage d'un petit robot),

- l'espace et la connaissance de la Terre.

Chaque thème possède son matériel spécifique et quelques références écrites. La connaissance de la Terre est abordée par le biais d'une sensibilisation à la météorologie. C'est bien-sûr cette dernière qui nous intéresse le plus ici.

Quand météorologie rime avec poésie (poème des élèves)

\section{Si j'étais...}

Si tu étais le vent, tu soufflerais fortement.

Si ce garçon était un tourbillon, il soulèverait le cochon.

Si tu étais la mousson, tu noierais les moutons.

Si j'étais le sirocco, je ne soufflerais que dans les pays très chauds.

Si j'étais le vent, je soufflerais moins souvent.

Si l'ouragan soufflait longtemps, il détruirait les cerfs-volants.

S'il y avait le sirocco, il détacherait les noix de coco.

Si le vent n'existait pas, le planeur ne volerait pas.

Si vous étiez la brise, vous voleriez au-dessus de la remise.

Si elle était la tornade, elle bousculerait le deltaplane.

Si vous étiez le vent, vous voyageriez à travers le temps.

Si tu étais l'ouragan, tu serais le roi des vents.

Si la bourrasque venait, je me cacherais sous mon masque.

Si j'étais le vent, le moulin tournerait longtemps.

Si jétais la tempête, je ne ferais aucun mal aux bêtes.

Si jétais la tornade, je ne pousserais pas mon camarade.

Si la rafale soufflait très fort, nous n'irions pas dehors.

Si tu étais un ouragan, tu serais très puissant.

S'il était une girouette, il serait une chouette.

Si vous étiez les alizés, vous aideriez à produire de l'électricité.

Si le vent le voulait, l'éolienne tournerait. 


\section{LES ACTIVITÉS EN MÉTÉOROLOGIE}

\section{La station météo}

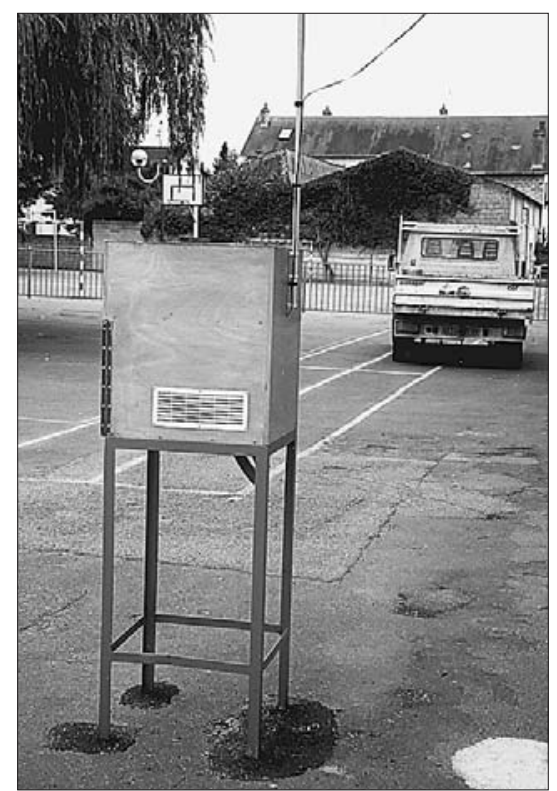

L'abri météorologique dans la cour de l'école.
Aborder la météorologie à l'école n'est pas chose facile du fait de la complexité des éléments qui entrent en ligne de compte. Il s'agit néanmoins pour nous de poursuivre une démarche scientifique et rigoureuse, tout en restant simple, en proposant des explications qui s'appuient sur des observations. Pour ce faire, le « coin météo » se compose de trois éléments.

Une petite station a été réalisée à partir d'un abri situé dans la cour de l'école ; dans l'abri, se trouvent un thermomètre à maxima et à minima, un baromètre anéroïde et un hygromètre. Ont été placés également dans la cour un pluviomètre et une manche à air fabriquée par des élèves. Ces instruments ont essentiellement pour but l'apprentissage de la lecture et du fonctionnement de chacun d'eux. Il nous paraît essentiel, en effet, de savoir lire chacun de ces appareils. Mais, lors d'observations régulières, cet exercice devient tellement contraignant qu'il a plutôt pour effet, chez les enfants, de venir contrarier les apports positifs de la découverte. La manipulation des instruments acquise, il est donc préférable d'utiliser le deuxième élément, la station automatique.

\section{Construire une manche à air}

La manche à air indique la direction et la force du vent.

\section{Matériel:}

- 1 morceau de $60 \mathrm{~cm}$ sur $160 \mathrm{~cm}$ de toile non tissée.

- du fil de fer rigide.

- 1 Emerillon (dans un magasin d'articles de pêche).

- de la colle néoprène (attention en l'utilisant)

-1 manche en bois.

-1 pointe de $6 \mathrm{~cm}$.

-1 tube de stylo-bille $(4 \mathrm{~cm})$.

- 1 bout de fil de fer très fin.

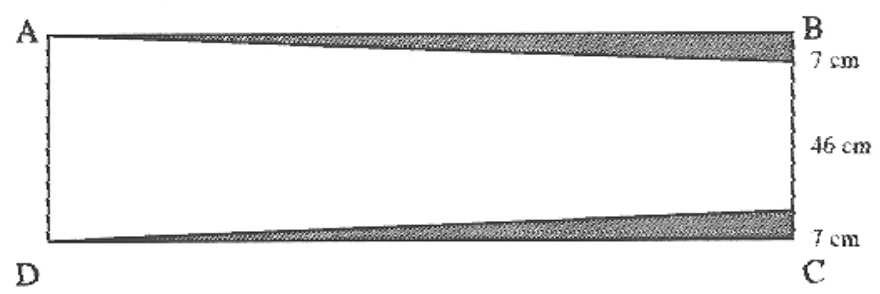

\section{Construction:}

- Découper la partie hachurée.

- Rabattre le côté AB sur le côté DC.

- Coller sur une bande de $2 \mathrm{~cm}$.

- Mettre en cercle $60 \mathrm{~cm}$ de fil de fer et rabattre le côté AD sur 2 cm par dessus se fil de fer. Coller.

- Plier en deux un morceau de fil de fer de $50 \mathrm{~cm}$ et l'attacher sur le cercle.

- Fixer l'émerillon.

- En haut du manche, enfoncer la pointe à travers le tube.

- Relier l'émerillon au manche avec le fil de fer fin en le faisant gasser à travers le tube en plastique.

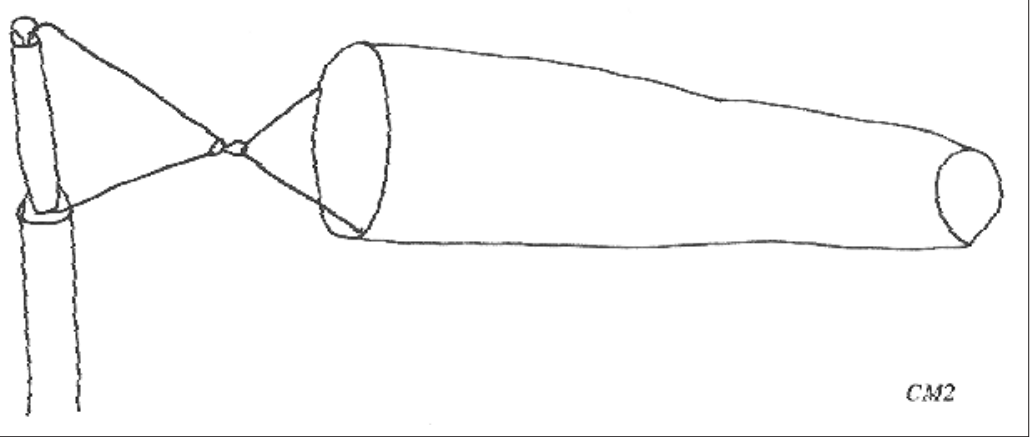




\section{La ministation automatique}

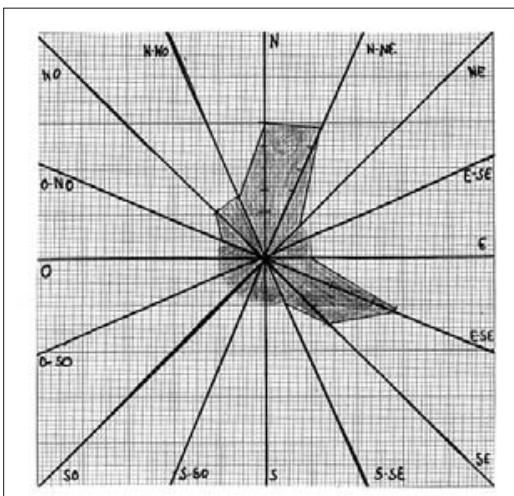

janvier 1996

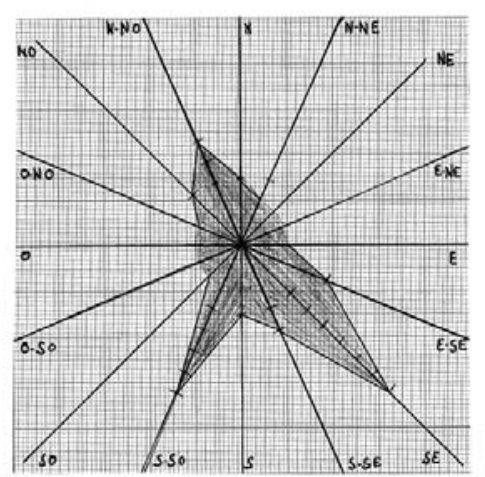

Février 1996

Figure 2 - Roses du vent, pour janvier et février 1996 à Sens, mesurées et tracées par les élèves.

\section{L'étude du vent}

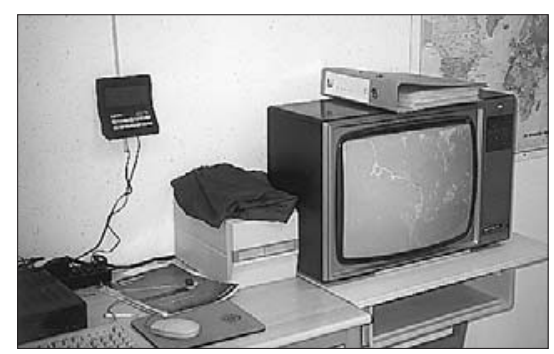

Fixé au mur du « coin météo », le boîtier de la station automatique.

À droite, le téléviseur relié au récepteur d'images satellitales.
Il s'agit d'une station Weather Monitor II de chez Davis Instruments. Elle est composée d'un petit boîtier de $15 \mathrm{~cm}$ x $13 \mathrm{~cm}$ qui permet de consulter sur son écran d'affichage toutes les données recueillies par les différents capteurs et accessoires, un câble téléphonique faisant le lien entre eux et le boîtier. Le kit de base comprend, outre le boîtier avec baromètre, thermomètre et hygromètre intérieurs, un anémomètre, une girouette et un capteur de température extérieur. Les autres accessoires sont en option. Pour notre part, nous avons ajouté un pluviomètre au kit de base.

Nous avons placé les capteurs de vent et de température sur le toit de l'école, mais le pluviomètre a été fixé sur le dessus de l'abri situé dans la cour, afin de pouvoir le nettoyer le cas échéant, le toit de l'école étant inaccessible.

L'ensemble du matériel, bien que léger, nous paraît solide. Il a été conçu pour être placé sur des bateaux et semble satisfaire aux exigences pour cet effet. En ce qui nous concerne, depuis un an qu'il est installé, il ne nous a posé aucun problème de résistance aux intempéries.

\section{Les différentes fonctions de la station automatique Weather Monitor II}

- Température intérieure.

- Température extérieure.

- Température mini-maxi.

- Direction du vent par paliers de 1 degré ou de 10 degrés.

- Vitesse du vent.

- Température fictive tenant compte de l'influence du vent.

- Pression atmosphérique (avec fonction mémoire permettant d'indiquer la tendance).

- Taux d'humidité intérieure.

- Taux d'humidité mini-maxi.

- Enregistrement des mini-maxi avec date et heure.

- Alarme de tendance barométrique pour tout changement supérieur à $0,5 \mathrm{~mm}, 1 \mathrm{~mm}$ ou 1,5 $\mathrm{mm}$ de mercure par heure.

- Pendule 12 ou 24 heures.

- Date.

- Une touche « Units » permet une lecture des mesures en système métrique ou en unités de mesure américaines.

Parallèlement, nous nous sommes procurés le logiciel Weatherlink qui existe en version PC et Macintosh. Un cordon, fourni avec le logiciel, relie le boîtier de la Weather Monitor II à l'ordinateur. Le logiciel est conçu en deux modules. Le premier permet de lire directement sur l'écran un bulletin instantané et de charger dans des fichiers journaliers toutes les données reçues et stockées par le boîtier. Le second permet de tracer à partir des données différentes sortes de courbes sur la journée, la semaine, le mois ou l'année, avec la possibilité de choisir les unités, les valeurs extrêmes des données et le type de courbes (points ou barres). Il est possible, en outre, de superposer deux types différents de courbes et de pouvoir ainsi observer le rapport qui peut exister entre deux types de données pour une même période, par exemple entre la température et la pression. Pour notre part, la classe a utilisé simultanément les relevés de vitesse et de direction du vent. Ainsi, les élèves ont pu construire des roses de vent mensuelles et déterminer les vents dominants (figure 2) ; toutes les journées de vent nul durant lesquelles l'ordinateur continuait de mettre en mémoire la direction du vent ont été éliminées.

Ce système automatique est évidemment très appréciable, puisqu'il permet des enregistrements réguliers, jour et nuit... y compris les jours de vacances ! L'exploitation des données est donc fiable. Dans notre cas, les roses de vent ont été tracées à partir des données de l'ensemble des jours de chaque mois, données chargées toutes les trente minutes, 24 heures sur 24. 


\section{Le récepteur d'images satellitales}

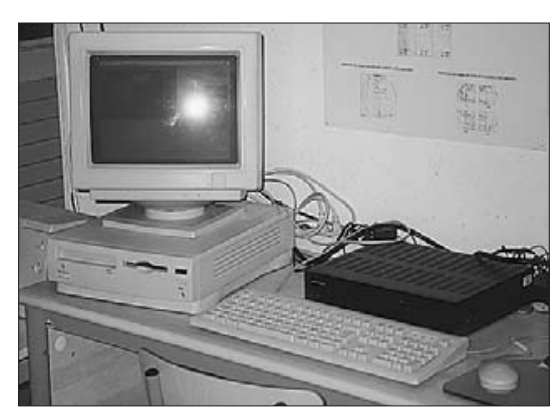

Le récepteur d'images satellitales Grundig (boîtier sombre) et le micro-ordinateur Macintosh du « coin météo ».
Il s'agit d'un récepteur Grundig MST 100 permettant de recevoir les images transmises par Météosat en mode Wefax (analogique). Nous l'avons placé sur un bureau afin de le raccorder, à la fois, à un récepteur de télévision et à notre micro-ordinateur Macintosh, qui possède une carte d'acquisition vidéo. Ainsi, nous pouvons enregistrer, conserver et animer des images reçues avec des manipulations excessivement simples.

L'installation individuelle se compose des éléments suivants :

- un récepteur satellite météo pour les deux canaux Météosat (1,691 et 1,6945 GHz), - une antenne de type Yagi de $120 \mathrm{~cm}$,

- un convertisseur situé directement sur l'antenne et alimenté à distance.

Parmi les caractéristiques du récepteur, citons :

- l'identification automatique du type d'image,

- le type d'image librement programmable (secteur géographique et canal spectral), - la fonction zoom,

- la réception d'une image simple, ou de six images dans chacune des deux banques d'images mémorisables, permettant donc d'obtenir des animations,

- la réception des images en noir et blanc ou dans trois gammes d'images colorisées (sur un téléviseur PAL).

L'appareil étant préprogrammé, il suffit donc de choisir le canal spectral et le secteur géographique pour que le récepteur stocke l'image dès qu'elle est envoyée par Météosat. Malheureusement, pour les images des satellites Goes et GMS, Eumetsat a changé leur format et celui-là ne correspond plus exactement à la préprogrammation. Il est néanmoins possible de recevoir ces images, mais cela nécessite une petite recherche. Par ailleurs, l'observateur doit se poster devant le récepteur s'il veut enregistrer certaines de ces images, car elles sont envoyées les unes à la suite des autres et chaque nouvelle image efface la précédente. Ces images sont cependant relativement rares dans une journée.

Les images reçues couvrent les différentes parties du globe avec possibilité d'animation sur l'Europe. La face de la Terre visible par Météosat est divisée en neuf secteurs, le secteur de l'Europe étant lui-même divisé en deux. Le continent américain est divisé en trois secteurs et l'Asie en quatre. Il est possible de recevoir les images dans les canaux infrarouge, visible et vapeur d'eau. Dans chacun de ces canaux, la cadence de réception est différente, les images infrarouges étant les plus nombreuses. Le récepteur capte également une mire, ainsi que le bulletin administratif (qui signale par exemple les heures de « ranging » durant lesquelles la réception est impossible).

La manipulation de l'appareil est assez simple et la qualité des images est correcte, même si elle n'atteint pas celle des images à haute résolution que l'on peut voir à la télévision. En contrepartie, la réception est gratuite et c'est un atout certain pour les écoles! En ce qui concerne le travail effectué avec les enfants, on peut dire que ce récepteur constitue un excellent outil pédagogique, compte tenu des innombrables pistes de travail qui sont offertes.
Figure 3 - Extrait du reportage sur les cyclones tropicaux réalisé par les élèves et image satellitale de l'ouragan Luis.
Les élèves ont ainsi pu réaliser un reportage sur les cyclones tropicaux (figure 3) à partir des images reçues à la fin de l'été et au début de l'automne 1995. Le fait de voir, pratiquement en direct, des images de la Terre a une grande importance pour l'intérêt des enfants : devant l'écran, ils ont la même « vue » que le satellite ; c'est du réel et ils peuvent comparer des images. Ainsi, les plus petits découvrent l'arrivée de la nuit sur notre continent, la rotondité de la Terre (qu'ils n'ont plus, hélas, l'occasion de découvrir sur les images des bulletins météo télévisés), les plus grands observent la naissance, la vie et la mort d'un cyclone, travaillent sur la géographie mondiale, sur la cartographie, le décalage horaire, etc. Les possibilités sont énormes, d'autant qu'en plus des images de l'Europe et de la moitié de la Terre qui fait face à Météosat, ce satellite relaie des images de l'Asie et de l'Amérique captées par les satellites GMS et Goes. 


\section{UN BALLON POUR L'ÉCOLE}

\section{Le fonctionnement du ballon}

\section{La construction du thermomètre}

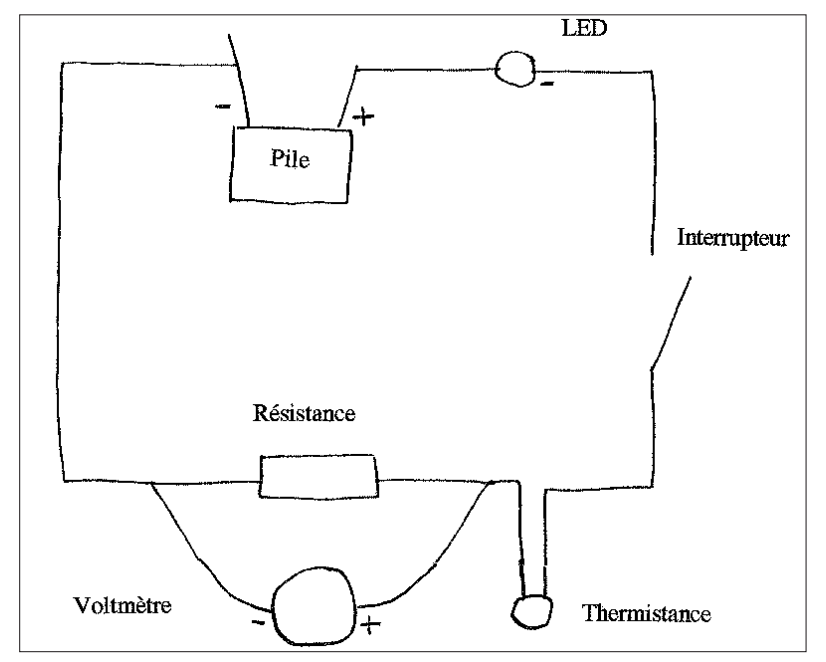

Outre l'emploi de ces instruments, qui a permis aux enfants de mener un travail de découverte et d'apprentissage sur le thème du vent, l'ensemble de la classe a aussi travaillé cette année sur le thème de la mesure de la température en altitude. Nous avons eu la chance de voir notre projet sélectionné dans le cadre de l'opération «Un ballon pour l'école » organisée par le Centre national d'études spatiales (CNES) et l'Association nationale sciences-techniques-jeunesse (ANSTJ). Voici ci-dessous le compte rendu qu'en ont effectué les élèves.

L'opération «Un ballon pour l'école » est organisée par l'ANSTJ et le CNES. Les élèves doivent imaginer une expérience scientifique, puis la réaliser pour l'envoyer dans une nacelle (qu'ils devront fabriquer); cette nacelle est accrochée à un ballon gonflé à l'hélium. Ce ballon peut monter à environ 40 kilomètres. Notre classe a été sélectionnée, au mois d'octobre 1995, pour participer à l'opération. Nous avons choisi de réaliser une expérience de mesure de la température en altitude, afin de répondre à la question : Comment varie la température avec l'altitude ?

Il fallait trouver un système qui mesure la température, qui l'enregistre et qui puisse donner l'altitude en même temps. Dans la nacelle, on ne peut pas placer un thermomètre ordinaire, car on a appris que la température en altitude pouvait descendre jusqu'à - $60{ }^{\circ} \mathrm{C}$. Premier problème : construire un thermomètre qui convient. Deuxième problème : comment enregistrer les données ?

Le ballon est gonflé à l'hélium (plus léger que l'air). C'est une enveloppe très légère et très fine. On y accroche un parachute qui va servir à ralentir la chute de la nacelle et un réflecteur radar qui permet aux avions et aux aiguilleurs du ciel de savoir où se trouve le ballon. Tout au bout du ballon, on place la nacelle qui
Figure 4 - Le parcours du ballon vu par les élèves.

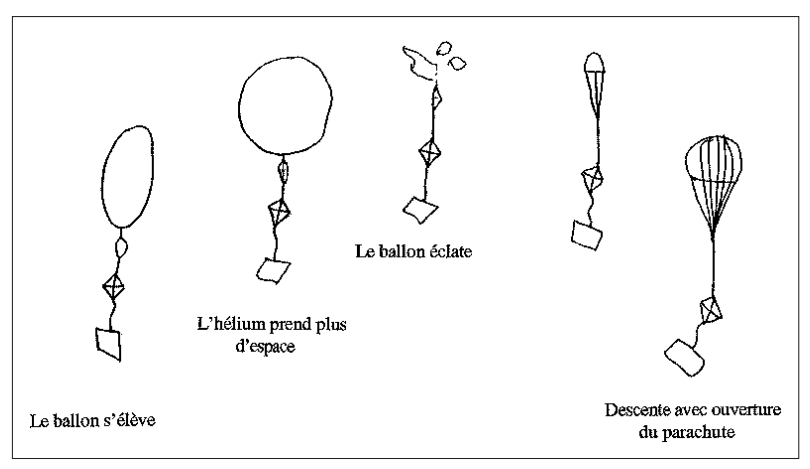
contient l'expérience scientifique. Après le lâcher, le ballon, plus léger que l'air, se met à monter. En montant, l'hélium prend plus de place car la pression atmosphérique diminue. Il arrive un moment où le ballon éclate, la nacelle descend et le parachute s'ouvre automatiquement.

À l'école, on a trouvé un vieil appareil (pyromètre) qui nous montre comment un morceau de cuivre se dilate à la chaleur et se rétracte en refroidissant. On a imaginé le système suivant : on a essayé de faire fonctionner notre appareil en prenant comme métal du cuivre et du plomb. Malheureusement, ce système n'a pas fonctionné car la dilatation n'était pas assez importante. Nous avons donc décidé de chercher une autre expérience en remplacement.

Dans Le détendeur (journal de l'opération « Un ballon pour l'école »), nous avons trouvé un schéma permettant de lire la température grâce à une thermistance (figure 5). Une thermistance est une résistance qui change de valeur avec la température. Quand elle change de valeur, l'aiguille du voltmètre se déplace sur le cadran. Il faut donc étalonner notre thermomètre pour savoir à quelles températures correspondent les positions de l'aiguille. Pour cela, on met la thermistance dans des endroits chauds ou froids (maison, congélateur, glaçons, etc.) pour inscrire, à chaque fois, les degrés sur le cadran. Nous avons réalisé ce montage qui fonctionne. Il faut faire attention à ne pas se tromper de sens (+ et -) pour la pile, le voltmètre et la LED. La LED sert de témoin pour savoir si le circuit est en marche quand la nacelle est fermée. 
Le dispositif de mesure de la température en altitude. À droite du coffret, le montage en Lego $\rightarrow$ pour la prise de vues automatique : à gauche, le voltmètre.

\section{Comment enregistrer les données?}

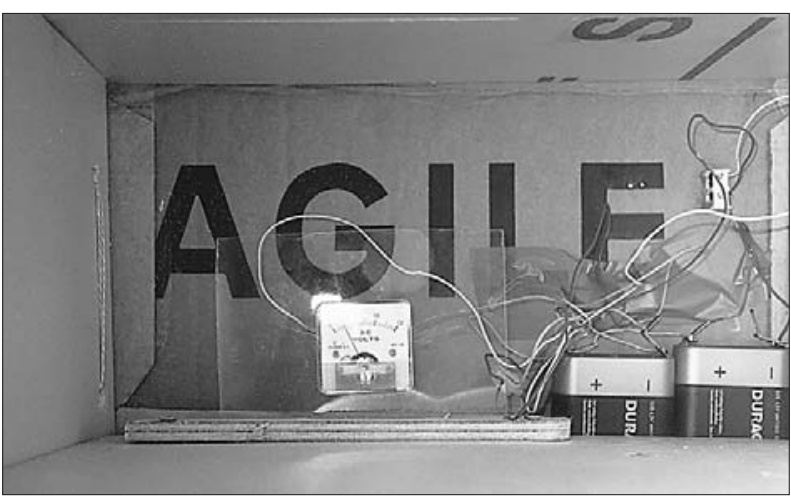

Le voltmètre et l'éclair du flash.

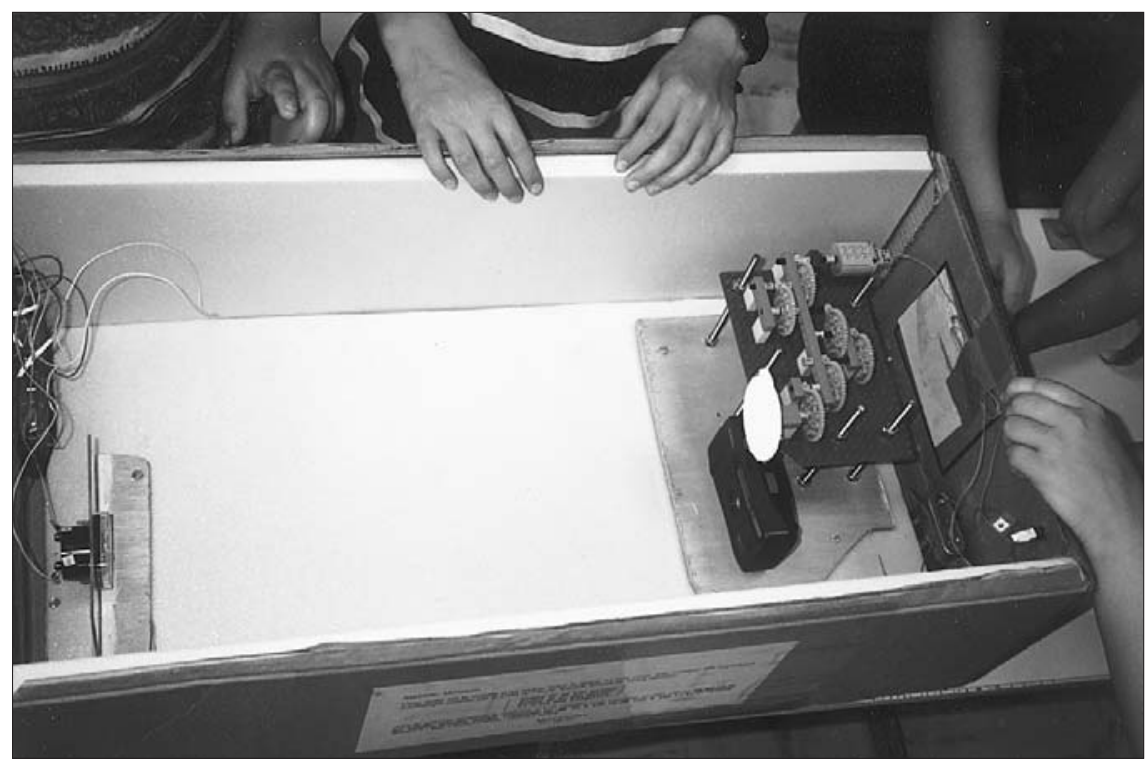

Avec le premier système, on avait pensé enregistrer les données sur un cylindre fixé sur un minuteur de cuisine. Comme la première expérience ne fonctionnait pas, on a décidé d'enregistrer les données par photo. Pour cela, il a fallu inventer un déclencheur. On sait que le ballon monte de 300 mètres en une minute. Si on prend des photos régulièrement, on peut savoir à quelle altitude se trouve le ballon. Il faut donc un système qui déclenche l'appareil photo tout seul, régulièrement, avec un temps égal entre chaque photo. Après plusieurs expériences, nous avons réalisé en Lego $\rightarrow$ un montage permettant à un morceau de carton en forme de 6 d'appuyer sur le déclencheur toutes les $3 \mathrm{mn} 20 \mathrm{~s}$.

Pour que le moteur tourne régulièrement (le froid peut le ralentir), on doit isoler la nacelle. La thermistance ne devra pas être isolée ; elle sera placée en dehors de la nacelle. Il a fallu aussi vérifier :

- si l'appareil photo pouvait être placé près du voltmètre et si les photos étaient nettes ; nous avons donc fait des photos à différentes distances ;

- si le flash n'éblouissait pas trop pour des photos de près ; on a fait des essais en mettant sur le flash différents caches en papier cristal car, sans cache, les photos étaient toutes blanches ; les meilleurs résultats ont été obtenus avec $50 \mathrm{~cm}$ entre l'appareil et le voltmètre, et avec un cache bleu plus un blanc par dessus.

\section{Problème de mathématiques !!!}

Notre système permet de prendre une photo toutes les $3 \mathrm{mn} 20 \mathrm{~s}$. Le ballon monte en moyenne de 300 m par minute.

Si nous mettons dans notre appareil une pellicule de 36 photos, jusqu'à quelle altitude va-t-on pouvoir relever les températures? Est-ce que ce système sera satisfaisant si le ballon monte jusqu'à $40 \mathrm{~km}$ ?

Pour que nos appareils soient isolés du froid, la nacelle est construite en polystyrène. On a éliminé le polystyrène expansé, car les bulles d'air prendraient plus de place en altitude à cause de la pression atmosphérique qui diminue et la nacelle exploserait. On a donc choisi du polystyrène de type « Roofmat ». Les morceaux de la nacelle sont collés. La nacelle est décorée. À l'intérieur, notre expérience est fixée sur une planche. On a placé en deux endroits le message ciaprès afin de pouvoir récupérer la nacelle. 
Madame, Monsieur.

Cette boüte est la nacelle d'un ballon lancé le mardi 11 juin 1996. Elle contient une expérience scientifique réalisêe par le CM2 de l'école Aristide-Briand de Sens (89100).

Prière de ne pas secouer.

Prierre de ne pas auurir.

Ce n'est pas dangereux.

Afin de pounair récupêrer le matêriel, SVP, wenillez nous contacter au plus vite au 86654237 de $8 \mathrm{~h} 30$ à $12 \mathrm{~h}$ et de $14 \mathrm{~h}$ à $18 \mathrm{~h}$ à l'école Aristide-Briand, rue Charles-Guerin. 89100 Sens.

Merci d'awance Les éteves

\section{Les autorisations}

\section{Les visites à l'école}

Le jour J

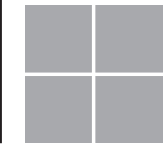

Remerciements

Ce projet pédagogique a pu être mené à bien grâce à l'aide et au soutien de la ville de Sens, de la Caisse des écoles de Sens, de l'Inspection académique de l'Yonne, de l'ANSTJ, du CNES et de la Société météorologique de France. Merci également à tous ceux qui, de façon individuelle, nous ont apporté leur concours. 


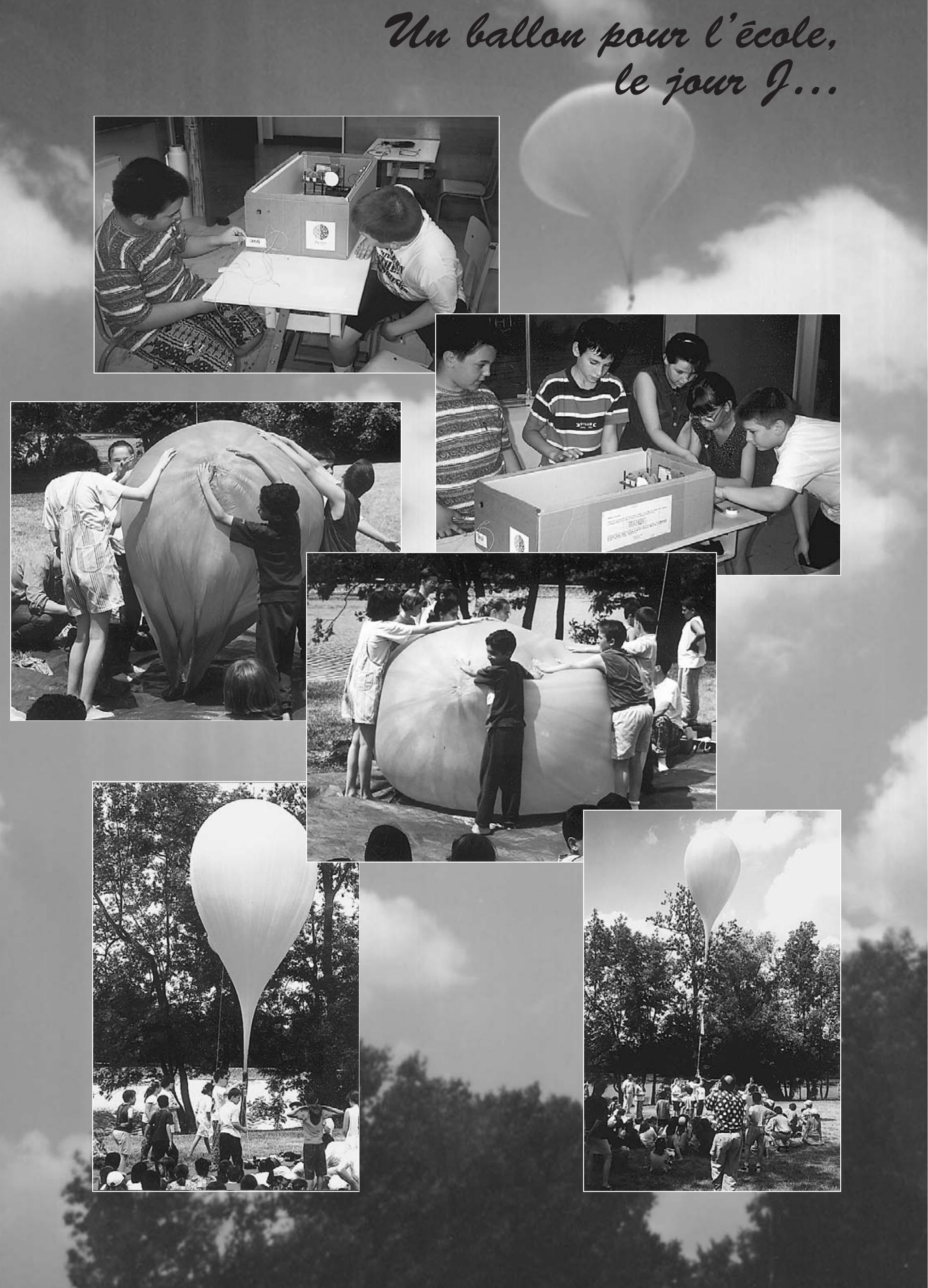


POUR EN SAVOIR PLUS

\section{PETITE BIBLIOGRAPHIE}

La station météo automatique peut se trouver à « La flotte française », 21, rue des Filles-du-Calvaire, 75003 Paris, ou encore chez « Générale Électronique Service », Z.I. de Savigny-le-Temple, Rue de l'Industrie, 77176 Savigny-le-Temple Cedex.

Prix TTC : la station, environ $3500 \mathrm{~F}$; le pluviomètre à vidange automatique, $850 \mathrm{~F}$; le capteur extérieur température-hygrométrie (point de rosée), $1450 \mathrm{~F}$; le logiciel Weatherlink, $1850 \mathrm{~F}$.

En ce qui concerne le récepteur Météosat Grundig MST 100, les lecteurs intéressés peuvent se renseigner auprès de M. Lascaray, Grundig France, à Rueil-Malmaison, téléphone : 0141392626 .

Secrets de la météorologie, Gallimard Jeunesse.

Météorologie, coll. «Les encyclopoches », Hachette.

Le ciel, l'air, le vent, coll. « Découverte Benjamin », Gallimard Jeunesse.

Vents et nuages, le temps qu'il fait, coll. « Les racines du savoir», Gallimard Jeunesse.

Le vent, par Jacques Bastian, Berger-Levrault.

Catastrophes naturelles - Les ouragans et les typhons, Gamma Jeunesse. Météo junior, CRDP du Limousin et Météo-France Haute-Vienne. Les nuages de A à Z, Éditions AAA et Météo-France.

Le temps qu'il fera, coll. «Les yeux de la découverte », Gallimard.

Le grand livre animé de la météo, par Francis Wilson, Héritage Jeunesse.

Le temps qu'il fait, coll. « Explorateurs en herbe », Seuil.

BT 874 : Les nuages et la météo. PEMF.

BT 954 : Les avalanches. PEMF.

BT 1017 : L'orage ; réchauffement de la Terre. PEMF.

BT 1042 : Les avalanches. PEMF.

BT 1064 : La neige. PEMF.

BTJ 80 : Il pleut, il neige. PEMF.

BTJ 345 : Le bulletin météo. PEMF. 